\title{
Laparoscopic repair of parastomal hernia
}

\author{
Xuefei Yang ${ }^{1 *}$, Kai He ${ }^{2 *}$, Rong Hua ${ }^{2}$, Qiwei Shen ${ }^{2}$, Qiyuan Yao ${ }^{2}$ \\ ${ }^{1}$ Department of Surgery, The University of Hong Kong-Shenzhen Hospital, the University of Hong Kong, Shenzhen 518053 , China; ${ }^{2}$ Hernia \\ Center, Department of General Surgery, Huashan Hospital, Shanghai Medical College, Fudan University, Shanghai 200040, China \\ *Those authors contributed equally to this work. \\ Correspondence to: Qiyuan Yao. Hernia Center, Department of General Surgery, Huashan Hospital, Shanghai Medical College, Fudan University, No. \\ 12 Middle Wulumuqi Road, Shanghai 200040, China. Email: Stevenyao@huashan.org.cn; 4683755@qq.com.
}

\begin{abstract}
Parastomal hernia is one of the most common long-term complications after abdominal ostomy. Surgical treatment for parastomal hernia is the only cure but a fairly difficult field because of the problems of infection, effects, complications and recurrence. Laparoscopic repair operations are good choices for Parastomal hernia because of their mini-invasive nature and confirmed effects. There are several major laparoscopic procedures for parastomal hernioplasty. The indications, technical details and complications of them will be introduced and discussed in this article.
\end{abstract}

Keywords: Parastomal hernia; laparoscopic repair; keyhole; sugarbaker; lap-re-do

Submitted Aug 29, 2016. Accepted for publication Dec 01, 2016.

doi: $10.21037 / \mathrm{atm} .2017 .02 .03$

View this article at: http://dx.doi.org/10.21037/atm.2017.02.03

Parastomal hernia is a bulge formed by intra-abdominal tissue or organs that protrude into the dilated artificial cavity surrounding the abdominal stoma after patients undergo abdominal ostomy. It is one of the most common long-term complications after abdominal ostomy. Its overall incidence is approximately $5 \%$ to $81 \%$, and the 1 -year postoperative incidence is approximately $30 \%$ to $50 \%$. Patients with parastomal hernia can present with skin irritation around the stoma, abdominal pain in the abdominal wall defect region, and difficulties with bowel movements. Severe cases might include incarcerated bowel loops that result in intestinal obstruction and necrosis. Parastomal hernia can reduce quality of life and represents a threat to the health and safety of patients $(1,2)$.

Surgery is the only cure for parastomal hernia. Local suture repair and repair with the relocation of the stoma have been associated with higher recurrence rates of hernia. Clinically, prosthetic material is commonly used to repair parastomal hernia. Over the past decade, the number of laparoscopic mesh repairs for parastomal hernia has gradually increased. Favorable results have been reported with regard to surgical safety, efficacy, and longterm follow-up assessments. Compared with traditional open surgical repairs for parastomal hernia, laparoscopic repair has certain advantages including its safe operation, postoperative rapid recovery, fewer complications, and lower recurrence rate. It has gradually become a widely accepted clinical practice (3-14).

\section{The laparoscopic repair of colostomy parastomal hernia $(15,16)$}

The Keyhole technique, the Sugarbaker technique, and the Sandwich technique are the common laparoscopic colostomy hernia repair maneuvers. We review these techniques below.

\section{Indications}

(I) Patients undergoing permanent colostomy;

(II) Parastomal bulge present and accompanied by abdominal distention or pain;

(III) Parastomal hernia results in leakage, difficult care of the colostomy bag, and ulceration in the stoma area;

(IV) The contents of the hernia sac are difficult to return, and a risk of intestinal incarceration exists;

(V) The large hernia sac affects the patient's appearance or normal daily activities. 


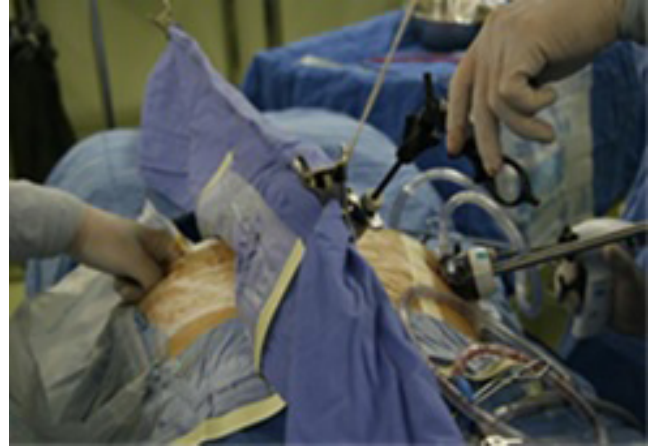

Figure 1 Preparation of the surgical fields.

\section{Contraindications}

(I) Tumor recurrence is identified during a preoperative evaluation or exploratory surgery;

(II) A preoperative examination reveals that patient is unable to tolerate general anesthesia or surgery because of poor heart and lung function or a blood coagulation disorder;

(III) A preoperative examination reveals that the patient has uncontrolled infections (e.g., those of the urinary tract, lung, or skin near the stoma).

\section{Preoperative preparation}

(I) Liquid diet begins 12 hours before surgery;

(II) Preoperative fasting starts, and bowel preparation is performed 6 hours before surgery;

(III) Prophylactic antibiotics should be given within 0.5 to 1 hour before the surgical incision is made.

\section{Anestbesia}

General anesthesia with endotracheal intubation.

\section{Preparation of the surgical field}

The abdominal surgical field and the surgical field near the stoma should each be disinfected three times. After the disinfected areas appear dry, a rectal catheter is placed into the colon through the stoma and can be used by the surgical assistant to help the surgeon identify the intestinal path during surgery. The stoma is covered by a piece of gauze and then sealed with an adhesive incise drape. A surgical towel divides the operative field into the right and left fields, and it is attached to the abdomen wall with the adhesive drape to separate the operative field from the potentially contaminated area around the stoma (Figure 1).

\section{Surgical position and trocar placement}

The patient is placed in the supine and Trendelenburg positions $\left(15^{\circ}\right)$ with a body tilt of $15^{\circ}$ to the right. The surgeon and the laparoscopic assistant stand on the right side of the patient, and the surgical assistant stands on the left side because the colostomy stoma is usually located in the lower left quadrant of the abdomen. A 3-cm incision is made at the anterior axillary line below the right costal margin; a $10-\mathrm{mm}$ (or 12-mm) trocar is then placed using an open technique or an optic trocar sheath. A $30^{\circ}$ laparoscope is placed into the abdominal cavity to inspect whether injury, bleeding, or abdominal adhesions are present. Under laparoscopic guidance, a $5-\mathrm{mm}$ trocar is placed into the right anterior axillary line above the anterior superior iliac spine (usually approximately $10-\mathrm{cm}$ below the first trocar) and in the midpoint between the umbilicus and the xiphoid process to serve as the operating port (Figure 2).

\section{Surgical procedures}

(I) Abdominal exploration and adhesiolysis. During abdominal exploration, the liver, omentum, and pelvis are inspected. It is also important to determine whether tumor recurrence or abdominal adhesions are present in the previous operative area; whether an occult incisional hernia is underneath the previous incision; or whether a concurrent inguinal hernia exists. At least $10 \mathrm{~cm}$ of the abdominal wall surrounding the stoma must be completely exposed for appropriate mesh placement. In most cases, an ultrasonic scalpel can be used for adhesiolysis. Sharp adhesiolysis should be performed with scissors to free the bowel loops from the abdominal wall in case of dense adhesions to avoid undetected burns of the bowel loops that can result in delayed postoperative intestinal fistula. Injured bowel loops must be repaired immediately;

(II) Return the hernia contents and free the bowel loop connected to the stoma. Damage-free grasping forceps are used to gently return the hernia contents. In cases of hernia sac adhesions, the adhesions should be separated, and then the contents can be returned. Given the requirements for securing the mesh via tacks (e.g., in the case of the Keyhole or Sandwich 




Figure 2 Surgical position and trocar placement. (A) Operating room layout; (B) trocar placement.

techniques), the bowel loop that connects to the stoma must be freed from the abdominal wall. Care should be taken to avoid injuring the bowel loop and mesenteric vessels, especially the latter because this injury can lead to intestinal necrosis and terminate the procedure. The path of these loops to the stoma can vary, and separation can easily injure the loops. Moreover, the injured bowel loop must be repaired with mesh, which increases the chance of infection, particularly when using a mesh containing e-PTFE. Therefore, the bowel loops within the hernia sac are not separated for the safety of the procedure. In fact, these bowel loops can be safely separated via the laparoscopy of an experienced hand. However, the colonic motility can push the loops separated from the sac to re-enter the previous hernia sac because they cannot be fixated appropriately in the abdomen cavity via full laparoscopy. Therefore, freeing the bowel loops from the hernia sac is meaningless;

(III) Measurement of the hernia ring size. Parastomal hernia must be completely exposed to measure the hernia ring size in the long and short dimensions;

(IV) Mesh selection. Most hernia rings range in size from 4 to $7 \mathrm{~cm}$. The mesh should be $15 \mathrm{~cm} \times 20 \mathrm{~cm}$, enough to cover the bowel loops to the stoma. In terms of a custom-made mesh for parastomal hernia repair, a larger mesh should be selected to allow for a 5 -cm overlap over the normal abdominal wall. If no custom-made mesh exists with a central opening, then the mesh can be incised at one side to create a $2-\mathrm{cm}$ multi-petal-shaped hole at the center (usually between $2 / 5$ and $3 / 5$ in the long dimension). An anti-adhesion mesh must be selected and inserted via a $12-\mathrm{mm}$ port into the abdominal cavity;

(V) Mesh fixation. The repair approach should be selected based on the length of the remaining bowel loop, the mesangial length, and the angle between the abdominal wall and the bowel loop. The Keyhole technique is recommended for cases with a larger angle and a shorter bowel loop; the Sugarbaker and Sandwich techniques are recommended for cases with a smaller angle and longer bowel loop. Spiral or absorbable tacks can be used to secure the mesh at 1to $2-\mathrm{cm}$ intervals.

In the Keyhole technique, the mesh (the incised opening toward the bowel loop) is placed around the bowel loop, and the mesh with the incised opening can be secured using tacks first. Then, the opposite and remaining mesh can be tacked based on the size of the bowel loop (the surgical assistant can insert his or her index fingers into the bowel loop to help control the size of the bowel loop). An overlap should be present at the incised opening of the mesh (Figure 3).

In the Sugarbaker technique, the mesh covers the segment of the bowel loop that is pushed to the lateral abdominal wall and the parastomal hernia defect. Using this approach, the bowel loop is pushed into the abdominal wall, and appropriate room between the mesh edge and the abdominal wall is left to allow the bowel loop to pass through. The mesh on both sides of this room should be tacked first, and then the mesh around the hernia ring and the mesh periphery can be tacked (Figure 4).

The Sandwich technique represents a combination of the Keyhole and Sugarbaker techniques. It includes three steps. Step 1 repairs 

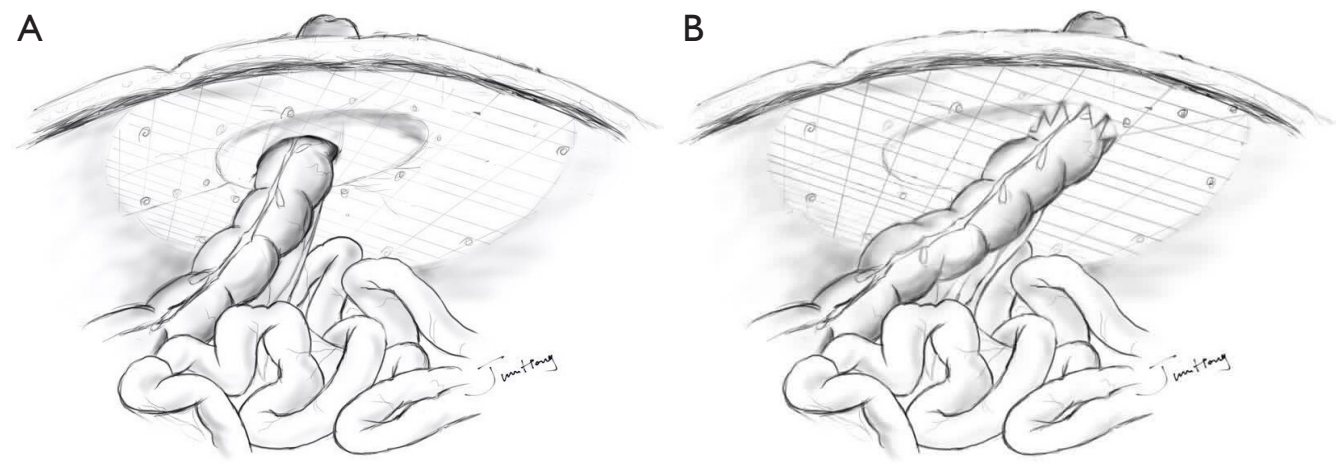

Figure 3 Keyhole technique.

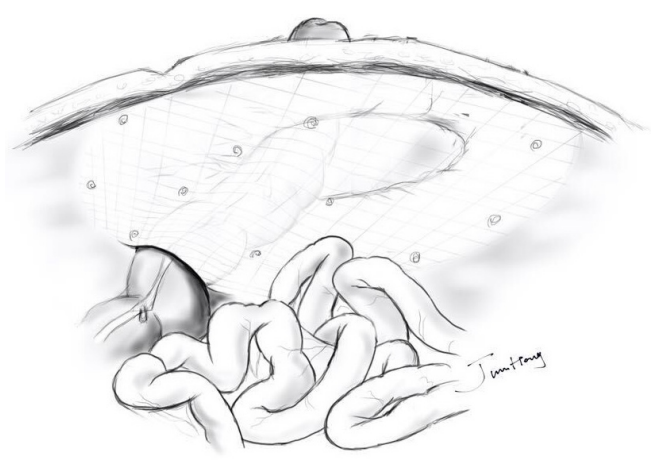

Figure 4 Sugarbaker technique.

the weakened area below the previous incision by placing a mesh; the anterior gap of the bladder is separated to expose the bilateral pubic arcuate ligaments and a portion of the lower edge of the hepatic round ligament. In step 2, a $15-\mathrm{cm}$ $\times 15-\mathrm{cm}$ anti-adhesion mesh with a $1-$ to $1.5 \mathrm{-cm}$ central opening is made for the bowel loop to pass through; the incised opening in the mesh is overlapped and then tacked to the abdominal wall (which is actually an application of the Keyhole technique for the defect repair). In step 3 , the Sugarbaker technique is employed: A $30-\mathrm{cm} \times$ $20-\mathrm{cm}$ anti-adhesion mesh is used to cover the stoma area and secure it to the abdominal wall by tacks. A section of the bowel loop is secured with appropriate room for the angle between the outside and inside mesh (Figure 5). Dr. Berger invented and first described this technique; he suggested that the abdominal wall weakness caused by surgical scarring should be repaired and reinforced with a combination of the Sugarbaker and Keyhole

\section{Precautions}

A strict aseptic technique is required because of the 



Figure 5 Dr. Berger's Sandwich technique for mesh fixation. (A) The fixation of the first mesh. This technique is similar to Keyhole technique; (B) the fixation of the second mesh. The lower edge can be pulled into the anterior gap of the bladder and fixed to the pubic arcuate ligament; (C) the fixation of the second mesh. The mesh covers the lateral bowel loop $(5-10 \mathrm{~cm})$. The technique is similar to Sugarbaker technique.

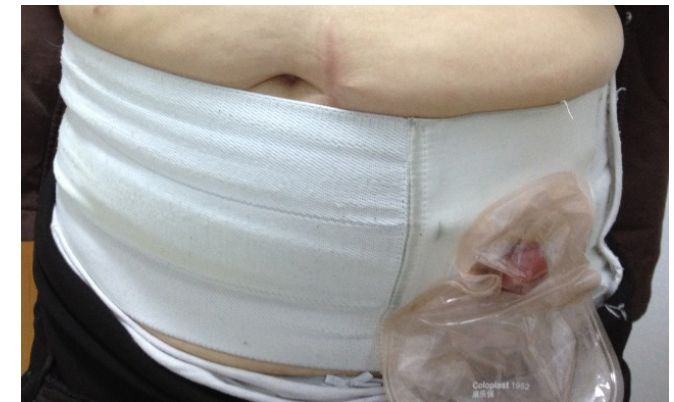

Figure 6 Customized abdominal support belt for stoma.

prosthetic mesh placement. When performing adhesiolysis, attention should be paid to a potential concealed bowel loop to carefully identify it and avoid potential bowel injury. The bowel loop and its mesenteric vessels should not be injured, especially the latter. Injury to these vessels can cause the stoma to re-construct and result in a failed surgery. If the bowel loop is injured, then the mesh repair should be delayed until 6 months after the injured bowel loop is repaired. The bowel loop within the hernia sac does not need to be separated to ensure the safety of this procedure. Moreover, the colonic motility can push the loops separated from the sac to re-enter the previous hernia sac to compromise the repair results and bowel movement function because these loops cannot be fixated in the abdomen cavity. Some surgeons have suggested closing the hernia ring for better repair results via laparoscopy. However, it is difficult to ideally close the hernia ring because of its tension and special position. Most types of anti-adhesion mesh are not transparent, which increases the difficulty of securing the mesh using tacks; therefore, the path of the bowel loops must be carefully identified, and bowel injury must be avoided. A suction drainage tube can help to inspect the peritoneal fluid properties and detect problems after surgery.

\section{Postoperative managements}

(I) Patients can be given a small amount of water 6 hours after surgery. A liquid diet can begin on the first day after surgery depending on the patient's status. A semi-liquid diet can start after passage of flatus. In addition, lactulose and other laxatives can be ordered as supportive therapy;

(II) The color of the stoma should be closely observed to assess its blood supply. Its drainage color and properties should be closely observed in cases with a drainage tube placement;

(III) Adequate analgesia;

(IV) Postoperative exercises in bed such as turns from side to side and leg elevation are recommended. Early ambulation is encouraged to prevent deep vein thrombosis, pulmonary embolism, and lung infection;

(V) An abdominal support belt is recommended for use with appropriate pressure for 3 months.

\section{Complications and managements (17)}

(I) Intraoperative complications. Laparoscopic surgery should be converted to open surgery under the following conditions: severe adhesion, difficulty identifying anatomic structures, suspicious injury of 


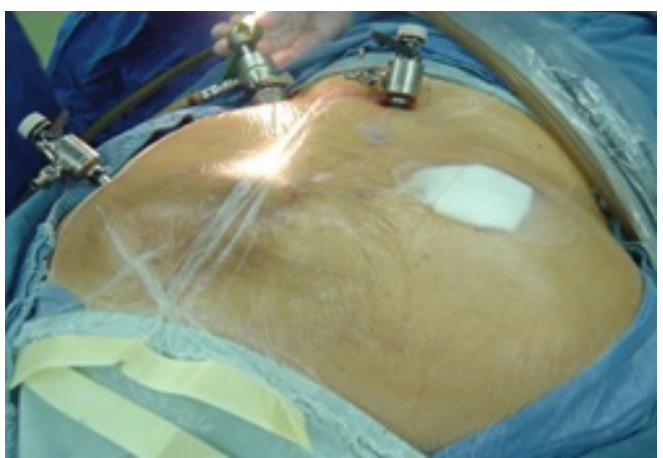

Figure 7 Surgical field preparation

adhered small bowel or stoma loop;

(II) Postoperative complications. (i) Undetected injury and perforation of the bowel loops can occur at the time of adhesiolysis and cause mesh infection. The mesh must be removed and the perforation fixed if the mesh contains e-PTFE or a severe abdominal infection is present. Therefore, the surgeons should be careful and composed during the operation; (ii) bleeding often occurs because of excessive adhesion separation, and it can effectively be avoided if an ultrasound scalpel is used for adhesion separation; (iii) one type of incomplete intestinal obstruction, adhesive small bowel obstruction, can be relieved via gastric decompression; another type, bowel (for the stoma) obstruction, can be resolved by combining anal catheterization and laxative therapy; (iv) the incidence of seroma is high after laparoscopic parastomal hernia repair because it is difficult to completely remove or close the sac via laparoscopy. Observation is allowed in cases with a small amount fluid that can be resolved spontaneously. If too much fluid is present, however, it should be removed via needle aspiration under ultrasound guidance to avoid accidental injury to the bowel loop and mesh contamination;

(III) Abdominal wall pain in the repaired area caused by the stimulation of the tacks and the mesh can be more salient and persist for a certain time period. Adequate analgesic treatment should be applied. Pressure dressing with an abdominal support belt not only relieves the pain but also effectively prevents recurrence. Patients should be advised to use it for 3 months as soon as they are able to get out of bed.

\section{The "Lap-re-Do" repair of the colostomy parastomal hernia}

Laparoscopic repair methods for colostomy parastomal hernia, including the Keyhole, Sugarbaker, and Sandwich techniques, use mesh bridging to cover the parastomal defect. None of these techniques address the hernia ring defect, subcutaneous sac, or the length of the bowel loop for the stoma. After surgery, colonic motility causes the bowel loops to re-enter the hernia sac and cause hernia recurrence. In addition, the stoma function improvement is not obvious, and the stoma appearance loses its symmetry at the previous stoma creation. The Sugarbaker and Sandwich procedures can achieve significant effects with regard to early parastomal hernia. In terms of late parastomal hernia with a large hernia sac, however, these two techniques can reduce the recurrence rate, but they do not improve the stoma function or abdominal appearance. Therefore, based on the laparoscopic repair of colostomy parastomal hernia, our center created a new technique defined as the "Lap-re-Do" technique in 2009 to manage hernia ring defect, subcutaneous sac, and the length of stoma bowel loop. The stoma is re-constructed in situ. This technique combines the advantages of a minimally invasive laparoscopic surgery and open surgery and has achieved good results. We describe this technique below.

\section{Indications, contraindications, preoperative preparation}

Same as the laparoscopic parastomal hernia repair described above.

\section{Preparation of surgical field}

The abdominal surgical field and the surgical field around the stoma should be disinfected three times. After the disinfected areas are dry, a rectal catheter is placed into the colon through the stoma so that the surgical assistant can help the surgeon identify the intestinal path during surgery. The stoma is covered by a piece of gauze and then sealed with an adhesive incise drape (Figure 7).

\section{Surgical positioning and trocar location}

Same as the laparoscopic parastomal hernia repair described above (Figure 8). 
A

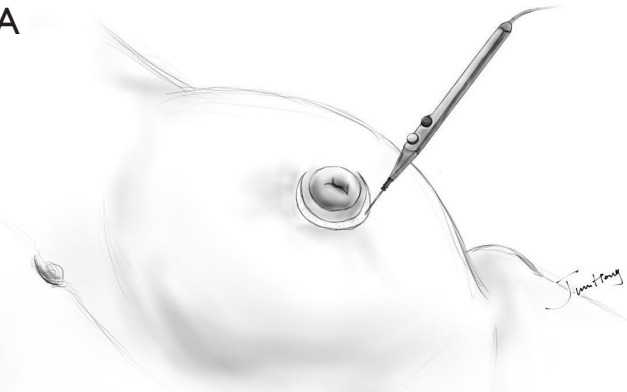

B



Figure 8 Annular incision for the stoma (A) in situ extended incision when the stoma is small; (B) in situ incision.

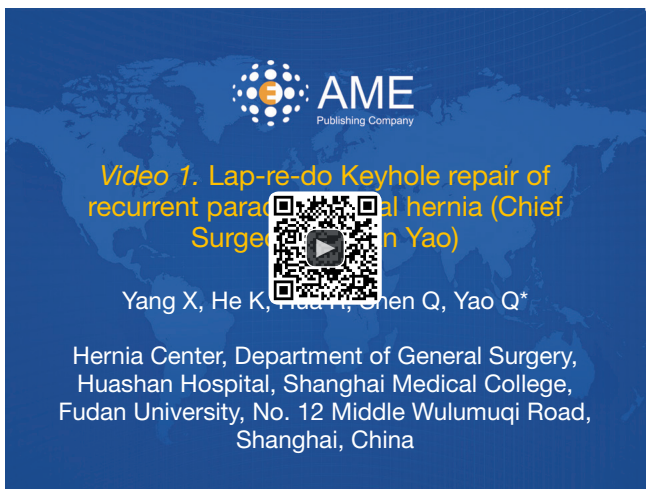

Figure 9 Lap-re-do Keyhole repair of recurrent paracolostomal hernia (Chief Surgeon: Qiyuan Yao) (18).

Available online: http://www.asvide.com/articles/1344

\section{Surgical procedures (Figures 9,10)}

(I) Abdominal exploration and adhesiolysis. During abdominal exploration, the liver, omentum, and pelvis are inspected. This exploration also seeks to determine whether tumor recurrence is present in the previous operative area, whether abdominal adhesions exist, and whether an occult incisional hernia is present underneath the previous incision. Ultrasonic dissector is recommended to perform adhesiolysis for omental

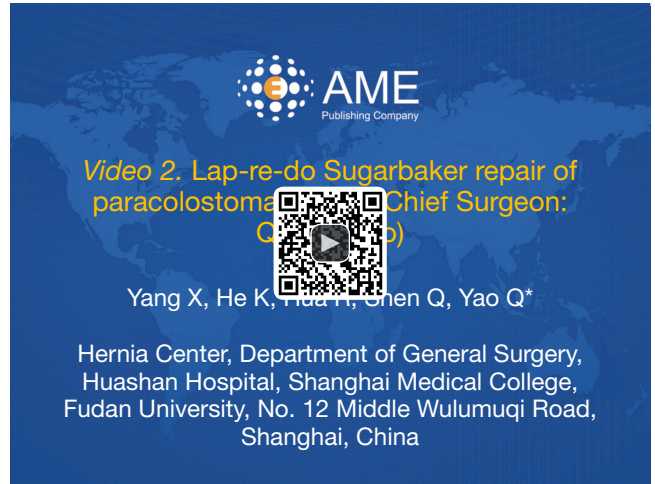

Figure 10 Lap-re-do Sugarbaker repair of paracolostomal hernia (Chief Surgeon: Qiyuan Yao) (19).

Available online: http://www.asvide.com/articles/1345

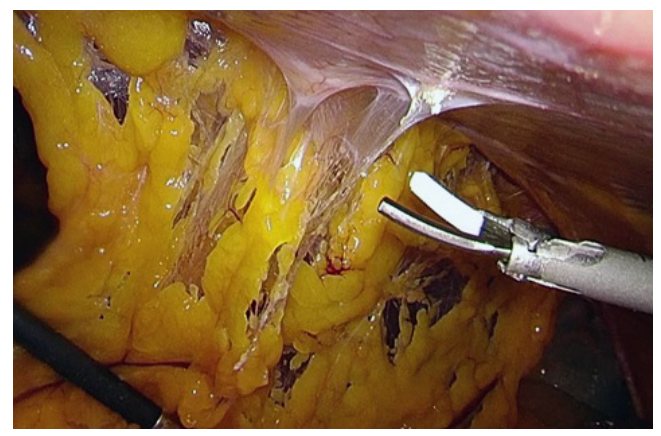

Figure 11 Ultrasonic dissector is used to separate the omental adhesions.



Figure 12 Scissors are used to separate bowel adhesions.

adhesions to avoid bleeding that can affect the visualization of the operative field. Sharp adhesiolysis should be performed with scissors to free the bowel loops from the adhesions to avoid the undetected burns of the bowel loops (Figures 11,12); 


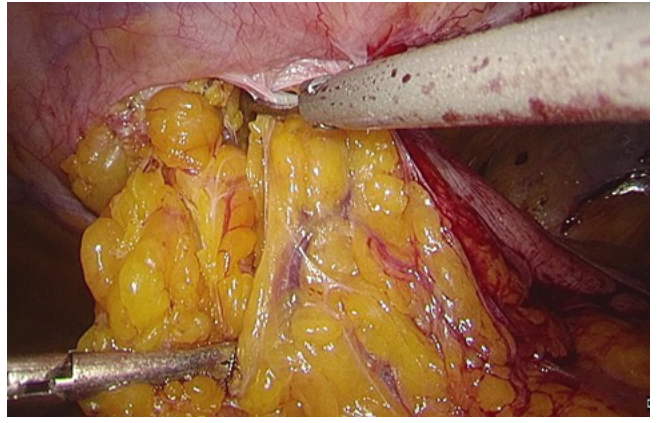

Figure 13 Ultrasonic dissector is used to separate adhesions within the hernia sac.

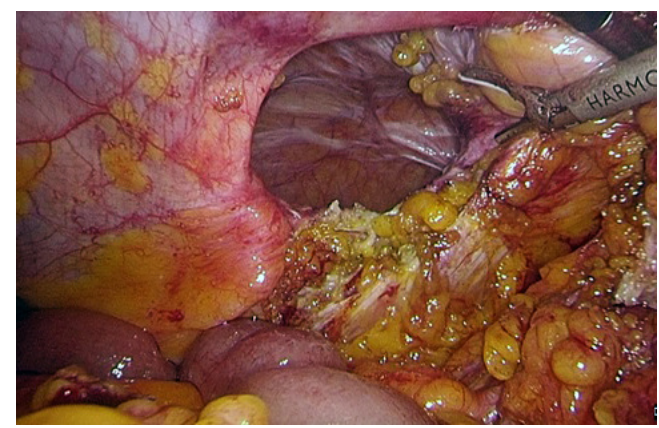

Figure 14 The bowel loop for the stoma is separated until reaching the subcutaneous layer.

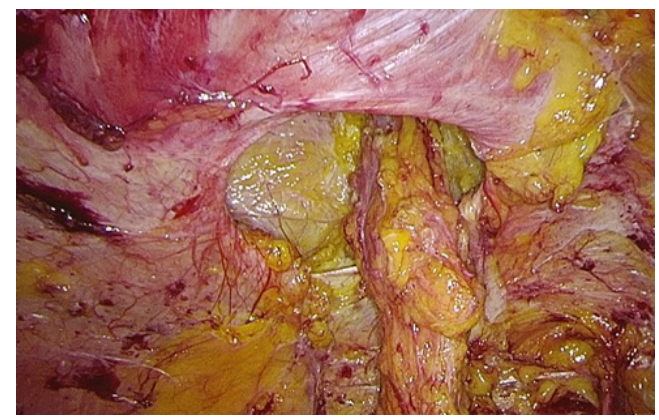

Figure 15 Exposure of the parastomal hernia ring.

(II) Return the hernia contents, free the bowel loop connected to the stoma, and measure the size of the hernia ring. Intestinal grasping forceps are used to gently return the hernia contents. Ultrasonic dissector is used to separate the adhesions within the parastomal hernia sac (Figure 13). The bowel loop for the stoma should be separated until reaching the subcutaneous layer (Figure 14). Care should be taken to avoid

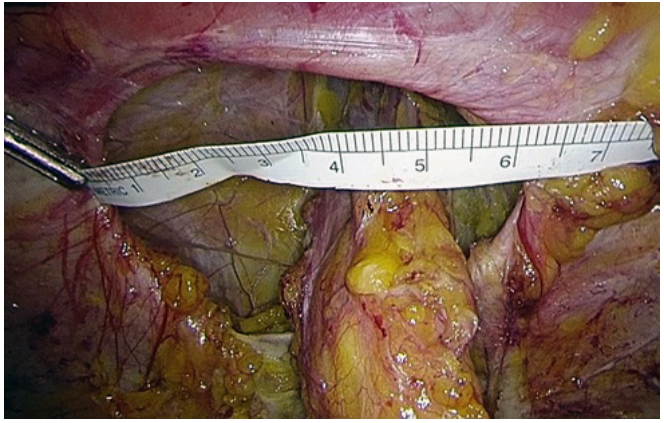

Figure 16 Measuring the size of the hernia ring defect.

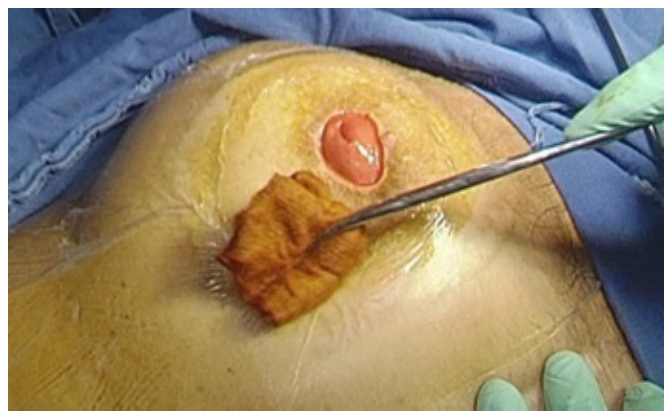

Figure $\mathbf{1 7}$ The re-disinfection of the area around the stoma before opening.

injuring the bowel loop and mesenteric vessels. The parastomal hernia must be, completely exposed (Figure 15) to measure the hernia ring size (Figure 16);

(III) Open the separated bowel loop. The area around the stoma is disinfected again (Figure 17). An annular incision is made along the proposed junction line of the stoma mucous and skin, and the bowel segment is brought through this incision out of the abdominal cavity. The distal end of this bowel segment is covered with sterile gloves and closed using a silk ligature (Figure 18). If the surgeons are concerned about injury to the small intestine, then the suspicious small intestine can be brought out of the abdominal cavity via this incision for inspection;

(IV) Mesh selection and placement. The repair procedure is selected based on the length of the bowel segment. Only the Lap-re-Do Keyhole technique can be used to repair the defect when the bowel segment is short. Either the Lap-re-Do Keyhole technique or the Lap-re-Do Sugarbaker technique can be used to repair the defect if the bowel segment is long. If the Lap-re-Do Keyhole technique is selected, then the specific Dynamesh-IPST 



Figure 18 A sterile glove is used to cover and close the distal end of the bowel segment.



Figure 19 The Dynamesh-IPST mesh is placed.

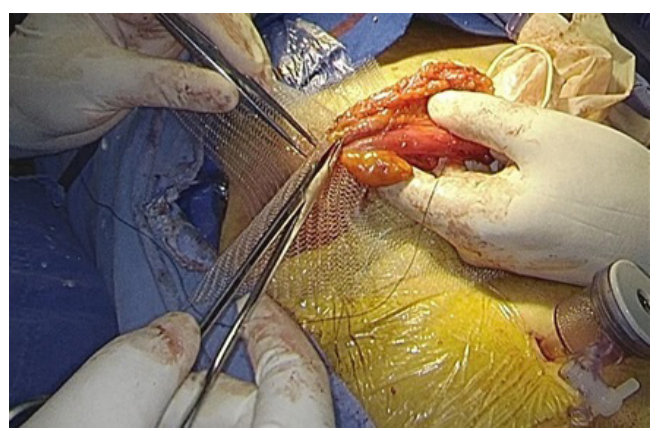

Figure 20 The mesh sleeve is sutured to the bowel.

mesh is recommended (Figure 19). Typically, its size is $15 \mathrm{~cm} \times 15 \mathrm{~cm} \times 2 \mathrm{~cm}$; the PVDF side should be facing the abdominal cavity, and the bowel segment should not be left in the abdominal cavity too long. After

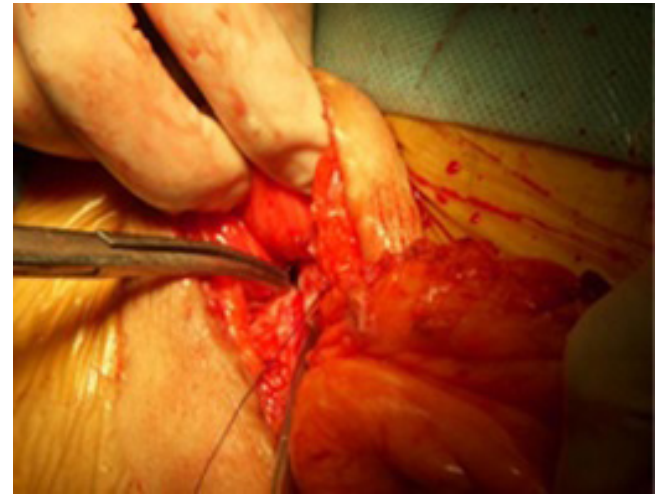

Figure 21 Closure of the previous parastomal hernia ring defect.

determining the length of the bowel segment, the mesh sleeve is sutured to the bowel using two layers of nonabsorbable sutures (Figure 20). Then, the mesh is placed into the abdominal cavity. Non-absorbable sutures are used to close the hernia ring, and a gap that can pass through one finger should be left adjacent to the bowel (Figure 21). If the Lap-re-Do Sugarbaker technique is used to repair the defect, an anti-adhesion mesh $(15 \mathrm{~cm} \times$ $20 \mathrm{~cm}$, transparent, lightweight, with large pores) should be placed into the abdominal cavity via a $12-\mathrm{mm}$ port after the hernia ring is closed;

(V) Fixation of the mesh under laparoscopic guidance. Using the Lap-re-Do Keyhole technique, the stoma site might need to be adjusted so that the mesh is 


\section{Page 10 of 16}

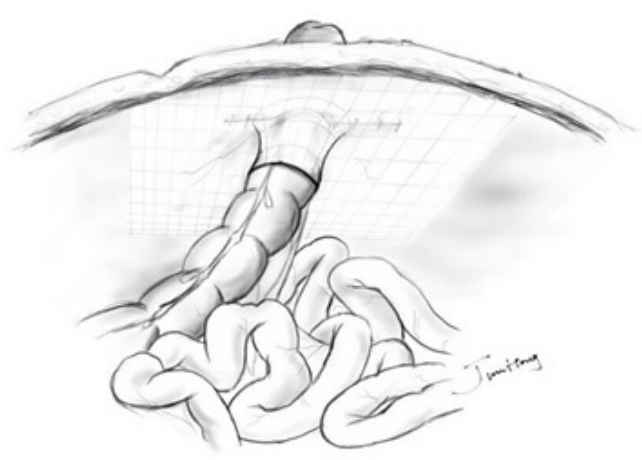

Figure 22 Schematic of the tacked mesh using the Lap-re-Do Keyhole technique.

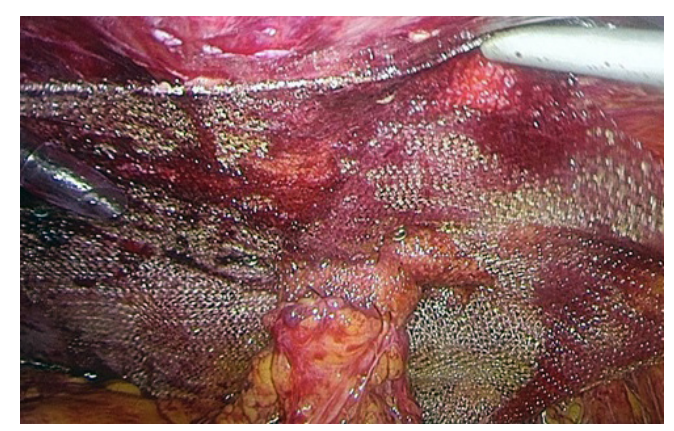

Figure 23 The tacked mesh using the Lap-re-Do Keyhole technique.

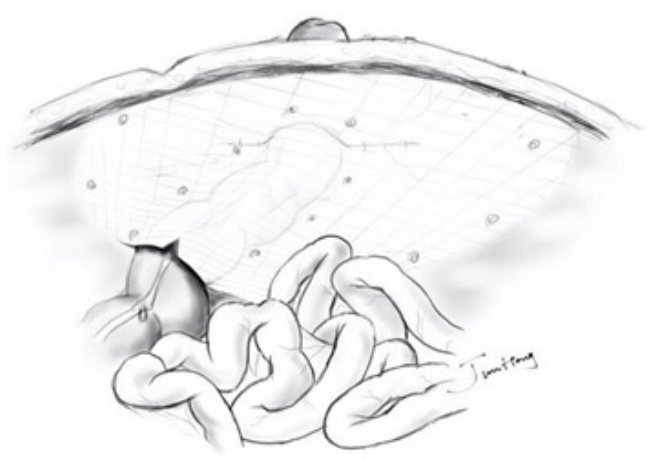

Figure 24 Schematic of the tacked mesh using the Lap-re-Do Sugarbaker technique.

located exactly underneath the abdominal wall. Spiral or non-absorbable tacks are used to secure the mesh around the bowel and along the edge of the mesh at 1 - to 1.5 -cm intervals (Figures 22,23). Using the Lapre-Do Sugarbaker technique, the bowel segment is
Yang et al. Laparoscopic repair of parastomal hernia



Figure 25 The tacked mesh using the Lap-re-Do Sugarbaker technique.

pushed into the lateral abdominal wall. The center of the mesh should align with the center of the stoma. The long axis of the mesh should be parallel with the longitudinal axis of the body. Spiral or non-absorbable tacks are used to secure the mesh around the bowel and along the edge of the mesh at $1-$ to $1.5-\mathrm{cm}$ intervals (Figures 24,25);

(VI) Drainage tube placement. The drainage tube is placed depending on the size and exudate of the surgical wound bed. The tube can be placed in the parastomal hernia repair area, passing over the pelvic floor and coming out via a 5 -mm port in the lower abdomen;

(VII) In situ re-constructed stoma. The subcutaneous sac should be excised completely, and the operative field bleeding should be controlled. Absorbable sutures are used to close the previous hernia sac. The placement of the drainage tube depends on the size of the previous hernia sac and the sac closure condition. The prolonged bowel is removed, and the stoma is reconstructed in situ (Figure 26). The colostomy bag is sealed over the stoma and dressed using the abdominal support belt.

\section{Key point analysis}

Laparoscopic Lap-re-Do repair including adhesiolysis, hernia content return, and mesh fixation is similar to laparoscopic parastomal hernia repair. However, the ultrasonic scalpel can be used in this procedure to free the bowel until it reaches the subcutaneous layer to reduce operation time and contaminant chances.

Mesh fixation is easier in the Lap-re-Do Keyhole technique than it is in the Lap-re-Do Sugarbaker technique. The mesh should not only cover the abdominal wall flat but 




B
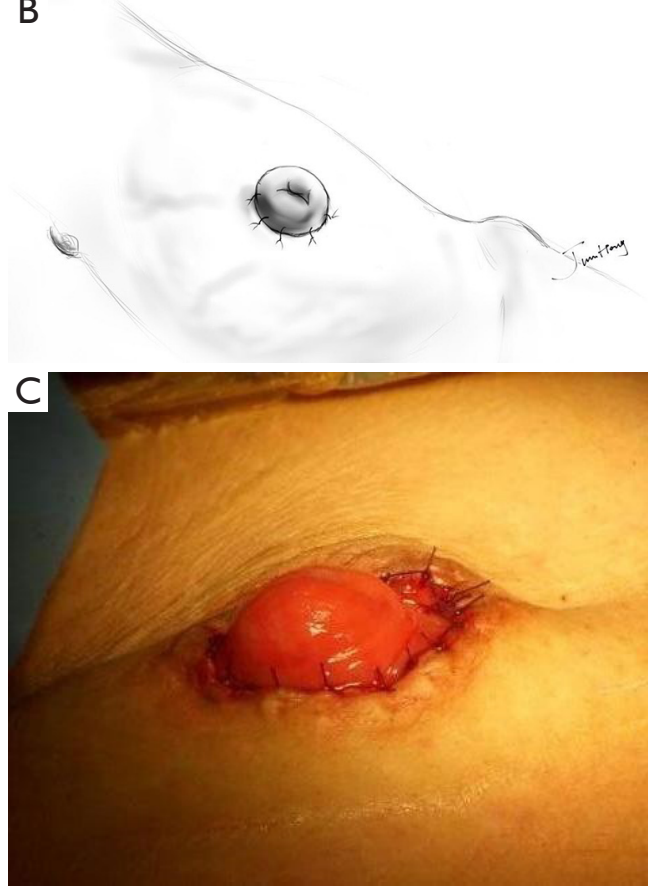

Figure 26 In-situ reconstruction of the stoma.

also leave a room for the bowel to pass through in the latter technique. The mesh around the stoma should be secured first. An anal catheter can be placed into the bowel. When necessary, the edge of the mesh that covers the bowel can be incised to $3 \mathrm{~cm}$ long to avoid bowel obstruction.

The Lap-re-Do technique, including the closure of the hernia ring, the excision of the subcutaneous hernia sac, the removal of the prolonged bowel, and the in-situ reconstruction of stoma, can return the stoma to its initial condition. This procedure not only effectively reduces the recurrence rate and prevents subcutaneous seroma but also improves abdominal appearance and postoperative bowel function. Moreover, the size of the re-constructed stoma can be modified regardless of whether the previous stoma opening is small or large. Thus, this technique can greatly improve the quality of life of patients.

\section{Precautions}

A strict aseptic technique is required for the Lap-re-Do technique because it involves the re-constructed stoma, the potential risk of operative field contamination, and the use of prosthetic materials. During surgery, the following managements should be performed appropriately to prevent infection in the area near the stoma: the stoma should be closed; the area around stoma should be disinfected; bowel injury should be prevented; the subcutaneous hernia sac should be closed and drained; and the proper anti-adhesion large-pore mesh should be selected.

\section{Postoperative managements}

The same techniques described above in the laparoscopic parastomal hernia repair section should be performed.

\section{Complications and managements}

(I) Intraoperative complications. If the surgeons are concerned about injury to the small intestine, then the small intestine can be brought out of the abdominal cavity via this incision for inspection. In terms of bowel injuries, a full-thickness rupture of the bowel might require laparoscopic repair to reduce contamination in the stoma area. Serosal injury alone can require the local repair or excision of the affected bowel depending on conditions observed during stoma creation. Laparoscopic surgery should be converted to open surgery in the case of severe adhesions and difficulty with identifying the anatomic structures;

(II) Postoperative complications. (i) Undetected injury of the bowel loops can occur at the time of adhesiolysis and should be identified carefully. An ultrasonic scalpel that can cause burns should not be used for dense adhesion. Re-operation is required to manage the undetected injury; (ii) bleeding is often due to excessive adhesion separation and can be effectively avoided via the use of an ultrasound scalpel to separate the omental adhesion; (iii) adhesive small bowel can be relieved via gastric decompression, whereas obstruction and bowel (for the stoma) obstruction can be resolved by combining an anal catheter with laxative therapy; (iv) hernia sac infection must be drained via tube placement, whereas mesh infection entails removal of the infected mesh; (v) the incidence of seroma is low because the sac wall is removed 
during the procedure. If there is too much fluid, however, then fluid removal via needle aspiration is recommended under ultrasound guidance to avoid accidentally injuring the bowel loops;

(III) Patients might not want to get out of bed because of postoperative abdominal pain. Inactivity can result in higher risks of deep vein thrombosis and pulmonary embolism. Patients should be encouraged to exercise early in the recovery process. Pain management and deep venous thrombosis prophylaxis should be applied.

\section{Laparoscopic repair of ileal conduit parastomal hernia}

The ileal conduit, also known as the Bricker procedure, is a common treatment of bladder cancer and various refractory cystitis. This procedure requires an ileal segment to be resected to form a substitute bladder and abdominal wall stoma. Approximately $5 \%$ to $65 \%$ of patients have ileal conduit parastomal hernia complications after this procedure and present with various symptoms. Most cases require reoperation.

\section{Indications}

(I) Gradually increased parastomal bulge after ileal conduit, accompanied by abdominal distension and pain;

(II) A parastomal hernia affects the tightness of the colostomy bag, resulting in urine leakage and difficult patient care;

(III) The contents of the hernia sac are difficult to return; abdominal pain is often present, and a risk of intestinal incarceration exists;

(IV) A large hernia sac greatly affects the patient's appearance and normal daily activities.

\section{Contraindications}

(I) Tumor recurrence is identified during preoperative evaluation or exploratory surgery;

(II) The preoperative examination reveals that the patient is unable to tolerate general anesthesia or surgery because of poor heart and lung function or a blood coagulation disorder;

(III) The preoperative examination reveals that the patient presents with uncontrolled infections, including those of the urinary tract, lung, and skin near the stoma.

\section{Preoperative preparation}

(I) A liquid diet begins 12 hours before surgery. Preoperative fasting starts, and the bowel is prepared 6 hours before surgery;

(II) Prophylactic antibiotics should be given within 0.5 to 1 hour before the surgical incision is made.

\section{Anesthesia}

General anesthesia with endotracheal intubation.

\section{Preparation of surgical field}

The abdominal surgical field and the surgical field near the stoma should be disinfected three times. After the disinfected areas appear to be dry, a Foley catheter $(10 \mathrm{~mL}$ of saline for balloon inflation) is inserted into the ileum pouch through the stoma. The stoma is covered by a piece of gauze and then sealed with an adhesive incise drape. A surgical towel divides the operative field into right and left fields, and the surgical towel is attached to the abdomen wall using the adhesive drape to separate the operative field from the potentially contaminated area around the stoma (Figures 27,28).

\section{Surgical positioning and trocar placement}

The patient is placed in the supine and Trendelenburg positions $\left(15^{\circ}\right)$ with a body tilt of $15^{\circ}$ to the left. The surgeon and the laparoscopic assistant should stand on the left side of the patient, and the surgical assistant should stand on the right side because the ileal conduit stoma is usually located in the lower right quadrant of the abdomen. A $3-\mathrm{cm}$ incision is made at the left anterior axillary line below the costal margin. A $10-\mathrm{mm}$ (or $12-\mathrm{mm}$ ) trocar is placed using an open technique or an optic trocar sheath. A $30^{\circ}$ laparoscope is placed into the abdominal cavity to inspect whether injury, bleeding, or both are present. Under laparoscopic guidance, a $5-\mathrm{mm}$ trocar is placed along the left anterior axillary line at the level of the umbilicus and at the midpoint between the umbilicus and the xiphoid process, serving as operating ports (Figure 29).

\section{Surgical procedures (Figure 30)}

(I) Abdominal exploration and adhesiolysis. During 


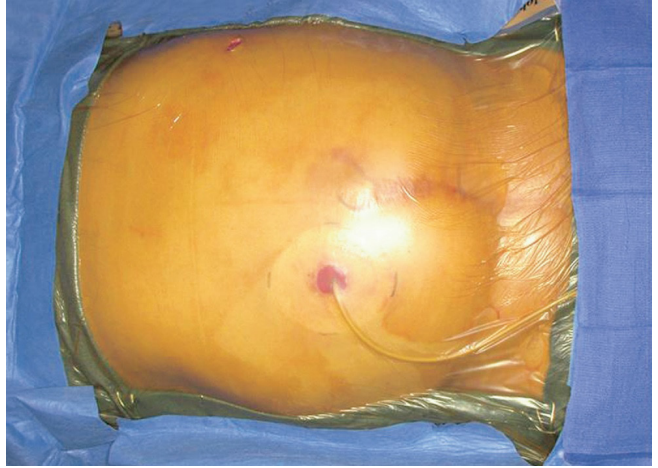

Figure 27 Surgical field preparation.



Figure 28 Segregation between the surgical field and the area around the stoma.


Figure 29 Surgical position and trocar placement. (A) Operating room layout; (B) trocar placement.

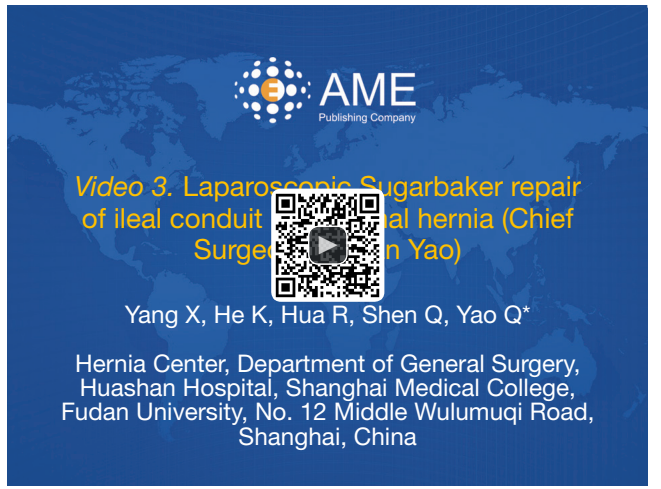

Figure 30 Laparoscopic Sugarbaker repair of ileal conduit parastomal hernia (Chief Surgeon: Qiyuan Yao) (20).

Available online: http://www.asvide.com/articles/1346

abdominal exploration, the liver, omentum, and pelvis are inspected. This exploration also seeks to determine whether tumor recurrence is present in the previous operative area, whether abdominal adhesions exist, and whether an occult incisional hernia is present underneath the previous incision. An ultrasonic scalpel is recommended for performing adhesiolysis for omental adhesions to avoid bleeding that can affect the visualization of the operative field. Sharp adhesiolysis should be performed with scissors to free the bowel loops from the adhesions to avoid the undetected burns of the bowel loops (Figure 31);

(II) Return the hernia contents. Laparoscopic damagefree grasping forceps are used to return the hernia contents (Figure 32), which usually include the small intestine or omentum. Gentle operations are required to avoid side injuries. The hernia contents are usually adhered to the hernia sac. Adhesiolysis is needed to reduce the hernia contents;

(III) Measurement of the hernia ring size. After the return 


\section{Page 14 of 16}

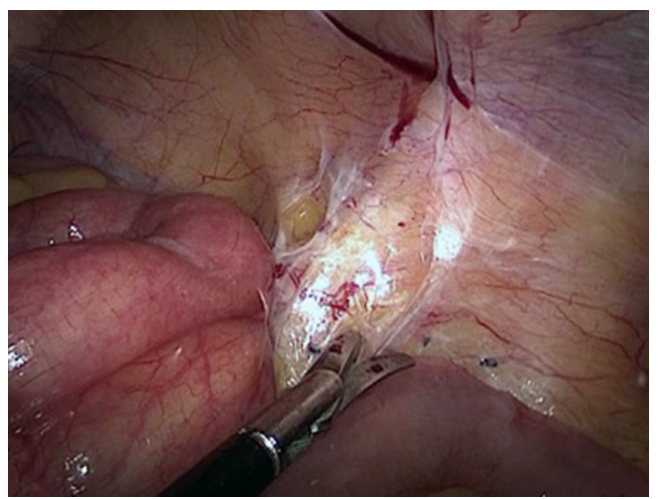

Figure 31 Sharp adhesiolysis of bowel adhesions with scissors.

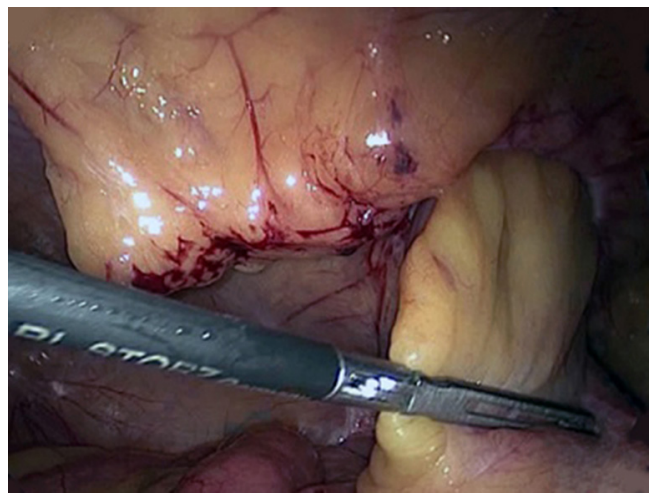

Figure 32 Reduction of the hernia contents.

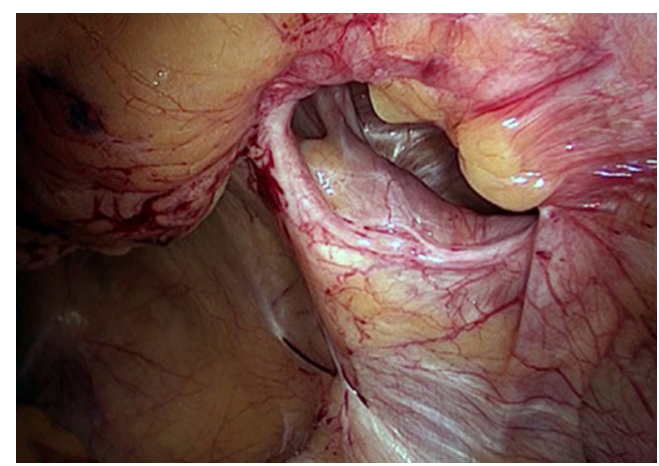

Figure 33 Exposure of the hernia ring.

of the hernia contents, the hernia ring is exposed (Figure 33). A $5-\mathrm{cm}$ abdominal area around the hernia ring must be completely separated to place the mesh and measure the hernia ring size (Figure 34);

(IV) Closure of the hernia ring. The bowel path should be

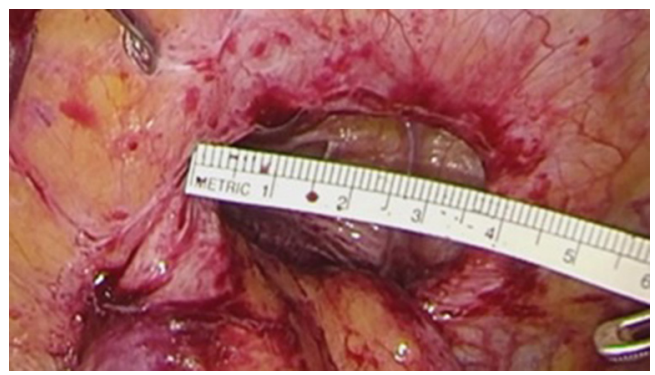

Figure 34 Measurement of the hernia ring size.

identified clearly. Non-absorbable sutures are used to close the hernia ring under laparoscopic guidance;

(V) Mesh placement and fixation via tacks. An appropriately sized anti-adhesion mesh should be selected to allow for a 5 -cm overlap of mesh to the normal abdominal wall. The anti-adhesion mesh is inserted via a $12-\mathrm{mm}$ port into the abdominal cavity. The anti-adhesion side faces toward the abdominal cavity. The Sugarbaker technique is used to repair the defect. Spiral or non-absorbable tacks are used to secure the mesh around the bowel and along the edge of the mesh at $1-$ to $1.5-\mathrm{cm}$ intervals (Figure 35);

(VI) Drainage tube placement. The drainage tube is placed depending on the size and exudate of the surgical wound bed. The tube can be placed in the parastomal hernia repair area, passing over the pelvic floor, and coming out via a 5 -mm port in the lower abdomen;

(VII) Under laparoscopic guidance, the trocars are withdrawn, and the pneumoperitoneum is removed. The $12-\mathrm{mm}$ trocar incision is closed. The remaining trocar incisions are closed using tissue glue. The colostomy bag is sealed over the stoma and dressed using the abdominal support belt.

\section{Key point analysis}

During adhesiolysis, the anatomic structure of the ileostomy bowel and its mesentery should be identified. The ileostomy bowel usually travels extraperitoneally. Therefore, the bowel loop and its mesenteric vessels must be carefully identified during abdominal surgery to avoid injury during adhesiolysis or tacked fixation. When necessary, an assistant should pull the Foley catheter to help the surgeon to identify the location of the bowel. 

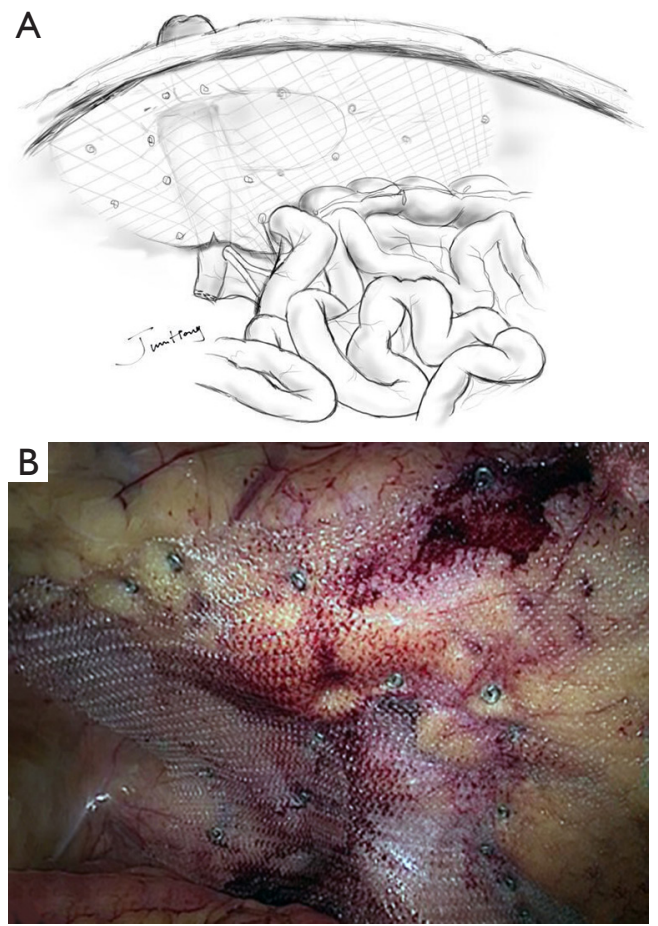

Figure 35 Mesh fixation using Sugarbaker technique.

\section{Precautions}

Tacked fixation of the mesh. The Sugarbaker technique can be used to secure the mesh. Care should be taken to avoid injuring the bowel loop (for the stoma) and its mesenteric and iliac vessels and to avoid tacking in the pain triangle region of the inguinal region.

\section{Postoperative managements}

The same management techniques described above in the laparoscopic parastomal hernia repair section apply here.

\section{Complications and managements}

(I) Intraoperative complications. Laparoscopic surgery should be converted to open surgery when severe adhesion is present. If the bowel loop (for the stoma) and the mesenteric vessels are injured, then the injured site should be repaired. In addition, a urological consultation should be ordered to inspect whether the original uretero-ileal anastomosis is intact;

(II) Postoperative complications. (i) The incidence of undetected injury to the adhesive bowel loops is approximately $0-9.1 \%$. This injury is a common complication during adhesiolysis. Sharp adhesiolysis should be performed with scissors for dense adhesions; instruments that can burn the tissue should not be used; (ii) bleeding is often caused by excessive adhesion separation and can effectively be avoided if an ultrasound scalpel is used for adhesion separation; (iii) the incidence of intestinal obstruction is approximately $6.3 \%$; this low incidence is because the ileal conduit is used for urine excretion; (iv) mesh infection is usually secondary to undetected bowel injury and requires re-operation. The anti-adhesion mesh containing e-PTFE should be avoided because it is made with smaller than 10-um pores; thus, macrophages and other immune cells cannot pass through to fight infection. Once this type mesh is infected, the infection can become intractable. Patients can present with persistent symptoms;

(III) Abdominal wall pain in the repair area caused by the stimulation of the tacks and mesh can be salient and persist for a certain time period. Adequate analgesic treatment should be applied. Pressure dressing with an abdominal support belt not only relieves pain but also effectively prevents recurrence. Patients should be advised to use it for 3 months as soon as they are able to get out of bed;

(IV) During laparoscopy, it is difficult to completely remove the sac wall, and seroma can form. Conservative observation is suggested for cases with small amount fluid or those without symptoms because this fluid can be resolved spontaneously. However, if too much fluid exists, it should be removed via needle aspiration under ultrasound guidance to avoid accidental injury to the bowel loop and mesh contamination.

\section{Acknowledgements}

Funding: Shenzhen government funding for scientific and technical research and development, Code: JCYJ20140414092023238.

\section{Footnote}

Conflicts of Interest: The authors have no conflicts of interest to declare. 


\section{References}

1. Moreno-Matias J, Serra-Aracil X, Darnell-Martin A, et al. The prevalence of parastomal hernia after formation of an end colostomy. A new clinico-radiological classification.

Colorectal Dis 2009;11:173-7.

2. Israelsson LA. Parastomal hernias. Surg Clin North Am 2008;88:113-25, ix.

3. Hansson BM, van Nieuwenhoven EJ, Bleichrodt RP, et al. Promising new technique in the repair of parastomal hernia. Surg Endosc 2003;17:1789-91.

4. Hansson BM, de Hingh IH, Bleichrodt RP. Laparoscopic parastomal hernia repair is feasible and safe: early results of a prospective clinical study including 55 consecutive patients. Surg Endosc 2007;21:989-93.

5. Hansson BM, Bleichrodt RP, de Hingh IH. Laparoscopic parastomal hernia repair using a keyhole technique results in a high recurrence rate. Surg Endosc 2009;23:1456-9.

6. Muysoms EE, Hauters PJ, Van Nieuwenhove Y, et al. Laparoscopic repair of parastomal hernias: a multi-centre retrospective review and shift in technique. Acta Chir Belg 2008;108:400-4.

7. Safadi B. Laparoscopic repair of parastomal hernias: early results. Surg Endosc 2004;18:676-80.

8. LeBlanc KA, Bellanger DE, Whitaker JM, et al. Laparoscopic parastomal hernia repair. Hernia 2005;9:140-4.

9. Sugarbaker PH. Peritoneal approach to prosthetic mesh repair of paraostomy hernias. Ann Surg 1985;201:344-6.

10. Berger D, Bientzle M. Laparoscopic repair of parastomal hernias: a single surgeon's experience in 66 patients. Dis Colon Rectum 2007;50:1668-73.

11. McLemore EC, Harold KL, Efron JE, et al. Parastomal

Cite this article as: Yang X, He K, Hua R, Shen Q, Yao Q. Laparoscopic repair of parastomal hernia. Ann Transl Med 2017;5(3):45. doi: 10.21037/atm.2017.02.03 hernia: short-term outcome after laparoscopic and conventional repairs. Surg Innov 2007;14:199-204.

12. Pastor DM, Pauli EM, Koltun WA, et al. Parastomal hernia repair: a single center experience. JSLS 2009;13:170-5.

13. Berger D, Bientzle M. Polyvinylidene fluoride: a suitable mesh material for laparoscopic incisional and parastomal hernia repair! A prospective, observational study with 344 patients. Hernia 2009; 13:167-72.

14. Halabi WJ, Jafari MD, Carmichael JC, et al. Laparoscopic versus open repair of parastomal hernias: an ACSNSQIP analysis of short-term outcomes. Surg Endosc 2013;27:4067-72.

15. Yao Q, He K. Choice of surgical treatments and skillful elements for parastomal hernia. Chinese Journal of Practical Surgery 2012;32:443-5.

16. Yao ZY, Chen H, Ding R, et al. Investigate the skillful elements in laparoscopic parastomal herniorraphy with meshes. Chinese Journal of Practical Surgery 2007;27:465-7.

17. Helgstrand F, Rosenberg J, Kehlet H, et al. Risk of morbidity, mortality, and recurrence after parastomal hernia repair: a nationwide study. Dis Colon Rectum 2013;56:1265-72.

18. Yang X, He K, Hua R, et al. Lap-re-do Keyhole repair of recurrent paracolostomal hernia (Chief Surgeon: Qiyuan Yao). Asvide 2017;4:038 .

19. Yang X, He K, Hua R, et al. Lap-re-do Sugarbaker repair of paracolostomal hernia (Chief Surgeon: Qiyuan Yao). Asvide 2017;4:039.

20. Yang X, He K, Hua R, et al. Laparoscopic Sugarbaker repair of ileal conduit parastomal hernia (Chief Surgeon: Qiyuan Yao). Asvide 2017;4:040. 